\title{
TRAVELLING WAVES SOLUTION FOR FRACTIONAL-ORDER BIOLOGICAL POPULATION MODEL
}

\author{
Hassan Khan ${ }^{1}$, Rasool Shah ${ }^{1}$, J.F. Gómez-Aguilar ${ }^{2,3, *}$, Shohib $^{1}$, \\ Dumitru Baleanu ${ }^{4,5,6}$ And PoOm Kumam ${ }^{7,8}$
}

\begin{abstract}
In this paper, we implemented the generalized $\left(\frac{G^{\prime}}{G}\right)$ and extended $\left(\frac{G^{\prime}}{G}\right)$ methods to solve fractional-order biological population models. The fractional-order derivatives are represented by the Caputo operator. The solutions of some illustrative examples are presented to show the validity of the proposed method. First, the transformation is used to reduce the given problem into ordinary differential equations. The ordinary differential equation is than solve by using modified $\left(\frac{G^{\prime}}{G}\right)$ method. Different families of traveling waves solutions are constructed to explain the different physical behavior of the targeted problems. Three important solutions, hyperbolic, rational and periodic, are investigated by using the proposed techniques. The obtained solutions within different classes have provided effective information about the targeted physical procedures. In conclusion, the present techniques are considered the best tools to analyze different families of solutions for any fractional-order problem.
\end{abstract}

Mathematics Subject Classification. 34A34, 35A20, 35A22, 44A10, 33B15.

Received December 11, 2020. Accepted March 19, 2021.

\section{INTRODUCTION}

Fractional-Order Partial Differential Equations (FPDEs) are frequently used in different physical phenomena in applied science such as biology, physics, engineering, systems identification, signal processing, control theory, fractional dynamics and finance. These applications of FPDEs in various research areas have attracted the researchers towards the subject of fractional calculus and particularly towards the study of FPDEs [10, 23,

\footnotetext{
Keywords and phrases: Extended $\left(\frac{G^{\prime}}{G}\right)$-expansion method, generalized $\left(\frac{G^{\prime}}{G}\right)$-expansion method, fractional-order biological population models, traveling wave solutions, Riemann-Liouville's derivative, complex transformation.

${ }^{1}$ Department of Mathematics. Abdul Wali Khan University Mardan (AWKUM), Pakistan.

2 CONACyT-Tecnológico Nacional de México/CENIDET, Interior Internado Palmira S/N, Col. Palmira, C.P. 62490, Cuernavaca Morelos, México

3 Consejo Académico,Universidad Virtual CNCI, Monterrey, México.

${ }^{4}$ Department of Mathematics, Faculty of Arts and Sciences, Cankaya University, 06530 Ankara, Turkey.

${ }^{5}$ Institute of Space Sciences, Magurele-Bucharest, Romania.

${ }^{6}$ Department of Medical Research, China Medical University Hospital, China Medical University, Taichung, Taiwan, Republic of China.

7 Center of Excellence in Theoretical and Computational Science (TaCS-CoE) \& Department of Mathematics, Faculty of Science, King Mongkut's University of Technology Thonburi (KMUTT), 126 Pracha Uthit Rd., Bang Mod, Thung Khru, Bangkok 10140, Thailand.

8 Department of Medical Research, China Medical University Hospital, China Medical University, Taichung 40402, Taiwan.

* Corresponding author: jgomez@cenidet.edu.mx
} 
24, 28]. In this regard, mathematicians have developed different techniques to solve FPDEs, which arise in various areas of applied science. Numerous numerical and analytical techniques have been developed $[13,17,19-$ $21,26,27,29,35]$ to investigate the solution of FPDEs. It is observed extensively that the analytical methods are difficult to apply for such problems and therefore it is considered a tough topic for mathematicians. In this connection some efficient and powerful analytical methods have been suggested such as the Hirota method [9], inverse scattering transform [1], the truncated Painleve expansion [14], the exp-function method [7], the Weierstrass elliptic function method [29], the simplest equation method [27], the Jacobi elliptic function method [19], ADM [35], the tanh-function method [9], the homogeneous balance method [1], the $\left(\frac{G^{\prime}}{G}\right)$-expansion method $[7,14]$ and so on.

Among these analytical methods, the most updated and reliable technique has been used, which is known as $\left(\frac{G^{\prime}}{G}\right)$-expansion method, for the solutions of FPDEs [15, 16, 18]. Initially, Wang et al. [6] have introduced the latest version of $\left(\frac{G^{\prime}}{G}\right)$-expansion method. In this approach, the traveling wave solutions can be written in the following form

$$
V(\xi)=\sum_{j=0}^{n} a_{j}\left(\frac{G^{\prime}}{G}\right)^{j},
$$

where $a_{j} \neq 0$.

Later on, Zhang et al. [22] have extended the simple $\left(\frac{G^{\prime}}{G}\right)$-expansion method for the traveling wave solutions of some non-linear PDEs. In the extended $\left(\frac{G^{\prime}}{G}\right)$-expansion method the solution can be expressed in the following form

$$
V(\xi)=\sum_{j=-n}^{n} a_{j}\left(\frac{G^{\prime}}{G}\right)^{j},
$$

where $a_{-n}$ and $a_{n}$ are not equal to 0 at same time.

Furthermore, this method is further modified for the solution of non-linear PDEs and FPDEs and can be written in the following form

$$
V(\xi)=\sum_{j=-n}^{n} a_{j}\left(l+\frac{G^{\prime}}{G}\right)^{j} .
$$

This method is called modified $\left(\frac{G^{\prime}}{G}\right)$-expansion method.

For all $\left(\frac{G^{\prime}}{G}\right)$-expansion methods mentioned above, the general solution of a linear second-order ordinary differential equation (ODE) with some constant coefficients have been used to solve FPDEs. The Maple software has been used to carry out the whole procedure by using an algorithm of $\left(\frac{G^{\prime}}{G}\right)$-expansion method. The obtained analytical solutions include periodic and solitary wave solutions. These traveling wave solutions are then presented in terms of trigonometric, hyperbolic and rational functions.

The aim of this article, is to use two modified $\left(\frac{G^{\prime}}{G}\right)$-expansion methods called extended $\left(\frac{G^{\prime}}{G}\right)$-expansion method and generalized $\left(\frac{G^{\prime}}{G}\right)$-expansion method for the analytical solutions of a $(2+1)$-dimensional degenerated fractional-order parabolic equation arising in spatial diffusion of biological populations. The general form of this equation is given as [12]:

$$
v_{t}^{\sigma}(t, x, y)=v_{x x}^{2}(t, x, y)+v_{y y}^{2}(t, x, y)+f(v), \quad x, y \in R, t \geq 0
$$


where $v$ represents the population density and $f$ denotes supply of populations due to deaths and births. Both $v$ and $f(v)$ are the functions of time $t$ and position $x, y$ in a region $\mathrm{R}$ and set out the diffusion of a biological species in $\mathrm{R}$. The function $v(x, y, t)$ exhibits the number of individuals in an unit volume at time $\mathrm{t}$ and position $x, y$. Over any subregion, the integral of $v(x, y, t)$ presents the total population of that subregion at any time t. The function $f(v)$ shows the average rate at that individuals are supplied per unit volume at $x, y$ by deaths and births straightforwardly. For $\sigma \rightarrow 1$, the following three examples of constitutive equations for $f(v)$ are:

1. $f(v)=c$ where $c$ is a arbitrary constant. This leads to Malthusian Law,

2. $f(v)=v\left(c_{1}-c_{2} v\right)$, where $c_{1}$ and $c_{2}$ are +ive constants. This leads to Verhulst Law,

3. $f(v)=-c v^{r}$, where $c \geq 0$ and $0<r<1$. It leads to Porous Media.

In present research work, we have considered the following particular forms of (1.1) i.e.:

$$
v_{t}^{\sigma}(t, x, y)=v_{x x}^{2}(t, x, y)+v_{y y}^{2}(t, x, y)+h\left(v^{2}-r\right)
$$

and

$$
v_{t}^{\sigma}(t, x, y)=v_{x x}^{2}(t, x, y)+v_{y y}^{2}(t, x, y)+v(1-r v)
$$

where fractional derivative $v_{t}^{\sigma}$ is described in the Riemann-Liouville derivative sense. This derivative operator is defined as:

$$
D_{t}^{\sigma} f(t)=\left\{\begin{array}{cc}
\frac{1}{\Gamma(1-\sigma)} \frac{\mathrm{d}}{\mathrm{d} t} \int_{0}^{t}(t-\phi)^{-\alpha}(f(\phi)-f(0)) \mathrm{d} \phi \\
& 1>\sigma>0, \\
\left(f^{(a)}(t)\right)^{(\sigma-a)}, & a+1>\sigma \geq a, a \geq 1 .
\end{array}\right.
$$

with the properties

$$
\begin{gathered}
D_{t}^{\sigma} t^{r}=\frac{\Gamma(1+r)}{\Gamma(1+r-\sigma)} t^{r-\sigma}, \\
D_{t}^{\sigma}(x(t) y(t))=y(t) D_{t}^{\sigma} x(t)+x(t) D_{t}^{\sigma} y(t), \\
D_{t}^{\sigma} x(y(t))=D_{g}^{\sigma} x[y(t)]\left(g^{\prime}(t)\right)^{\sigma} .
\end{gathered}
$$

Before the present research work, the mathematicians have used various techniques to solve biological population models. In this respect, Samia Bushnaq et al. [33] have extended the optimal homotopy asymptotic method (OHAM) to solve fractional-order biological population models. Fatemeh Shakeri and Mehdi Dehghan have found the numerical solution of some biological population models using He's variational iteration method (VIM) [12]. El-Sayed et al. have solved four biological problems with the help of ADM [4]. Baleanu et al. have investigated the biological models' solution by using a simple $\left(\mathrm{G}^{\prime} / \mathrm{G}\right)$-expansion method [31].

In this paper, two types of $\left(\frac{G^{\prime}}{G}\right)$-expansion methods have been used for the solutions of equations (1.2) and (1.3). It is concluded that the present study provides more families of traveling waves solutions and therefore analyze the physical problems in more directions as previously done by other methods.

The rest paper structure is as follows: Section 2 provides the methodology of the proposed method; in Section 3, analytical solutions of some selected biological population models are presented; some graphs of solution functions and discussion upon our study are presented in Section 4. Finally, a brief conclusion has been listed. 


\section{The $\left(\frac{G^{\prime}}{G}\right)$-EXPANSION METHOD}

In this section, the mechanism of $\left(\frac{G^{\prime}}{G}\right)$-expansion method for the solutions of FPDEs is presented. For this purpose, we consider the general non-linear FPDE given as:

$$
Q\left(v, D_{t}^{\alpha} v, D_{x_{1}}^{\beta} v, D_{x_{2}}^{\gamma} v, D_{x_{3}}^{\delta} v, v D_{x_{1}}^{\alpha} v \ldots\right)=0, \quad 0<\alpha, \beta, \gamma, \delta \leq 1,
$$

where $v$ is an unknown function of $x_{1}, x_{2}, x_{3}, \ldots, x_{n}$ and $t, Q$ is a polynomial in $v$ and its fractional partial derivatives. To solve (2.2), the following steps are taken:

i. First, a variable transformation called complex transformation given as [32]:

$$
\begin{aligned}
& v\left(t, x_{1}, x_{2} \cdots x_{n}\right)=v(\varphi), \\
& \text { where } \varphi=a \frac{t^{\alpha}}{\Gamma(\alpha+1)}+b \frac{x_{1}^{\beta}}{\Gamma(\beta+1)}+\cdots+\varphi_{0},
\end{aligned}
$$

where $a, b, \ldots, \varphi_{0}$ are arbitrary constants, is applied.

ii. This transformation converts (2.2) into a non-linear ODE of the form:

$$
P\left(V, V^{\prime}, V^{\prime \prime}, V V^{\prime}, \ldots\right)=0
$$

where the derivatives of $V$ in (2.3) are w. $\mathrm{r}$.t $\varphi$. If possible, (2.3) can be integrated w.r.t $\varphi$ one or more time.

iii. In third step we suppose $\frac{G^{\prime}}{G}$ solution for (2.3). This solution is expressed in the following cases:

Case 1: The extended $\frac{G^{\prime}}{G}$-expansion method represents the following series form solution:

$$
V(\varphi)=\sum_{i=-n}^{n} c_{i}\left(\frac{G^{\prime}(\varphi)}{G(\varphi)}\right)^{i}
$$

Case 2: The generalized $\frac{G^{\prime}}{G}$-expansion method represents the following series form solution:

$$
V(\varphi)=\sum_{i=-n}^{n} c_{i}\left(d+\frac{G^{\prime}(\varphi)}{G(\varphi)}\right)^{i}
$$

where $c_{i}^{\prime} s$ and $d$ are constants to be determined later. The positive integer $n$ present in equations (2.4) and (2.5) is called balance number and can be calculated by taking the homogenous balance between the nonlinear terms and the highest order derivatives terms in equation (2.3). More precisely the balance number can be found by using the following formulae [27]:

$$
\begin{aligned}
& D\left(\frac{d^{q} V}{\mathrm{~d} \varphi^{q}}\right)=n+q \\
& D\left(V^{p}\left(\frac{d^{q} V}{\mathrm{~d} \varphi^{q}}\right)^{s}\right)=n p+s(q+n),
\end{aligned}
$$

where $D$ denotes degree of $V(\varphi)$ i.e. $D[V(\varphi)]=n$ while $p, q$ and $s$ are positive integers.

The function $\mathrm{G}(\varphi)$ present in equations (2.4) and (2.5), satisfies the following second-order linear ODEs:

$$
G^{\prime \prime}(\varphi)+\lambda G^{\prime}(\varphi)+\mu G(\varphi)=0
$$


where $\lambda$ and $\mu$ are arbitrary constants. By the general solution of equation (2.7) we have [27]:

$$
\left(\frac{G^{\prime}}{G}\right)= \begin{cases}\frac{\sqrt{D}}{2} \frac{C_{1} \sinh \left(\frac{\sqrt{D}}{2} \varphi\right)+C_{2} \cosh \left(\frac{\sqrt{D}}{2} \varphi\right)}{C_{1} \cosh \left(\frac{\sqrt{D}}{2} \varphi\right)+C_{2} \sinh \left(\frac{\sqrt{D}}{2} \varphi\right)}-\frac{\lambda}{2} & D>0 \\ \frac{\sqrt{-D}}{2} \frac{-C_{1} \sin \left(\frac{\sqrt{-D}}{2} \varphi\right)+C_{2} \cos \left(\frac{\sqrt{-D}}{2} \varphi\right)}{C_{1} \cos \left(\frac{\sqrt{-D}}{2} \varphi\right)+C_{2} \sin \left(\frac{\sqrt{-D}}{2} \varphi\right)}-\frac{\lambda}{2} & D<0 \\ \frac{C_{2}}{C_{1}+C_{2} \varphi}-\frac{\lambda}{2} & D=0,\end{cases}
$$

where $D=\lambda^{2}-4 \mu$ while $C_{1}$ and $C_{2}$ are arbitrary constants.

iv. According to the method, (2.4) or (2.5) with the help of (2.7) is substituted in (2.3) which provides a polynomial in $\left(\frac{G^{\prime}(\xi)}{G(\xi)}\right)$.

v. Equating all coefficients of the same powers of $\left(\frac{G^{\prime}(\xi)}{G(\xi)}\right)$ in the subsequent polynomial to zero provides a system of non-linear algebraic equations in $c_{i}^{\prime} s, \lambda$ and $\mu$.

vi. The resultant system is then solved by using Maple software which determine the values of $c_{i}^{\prime} s, \lambda$ and $\mu$. By putting all obtained values of unknown coefficients in (2.4) or (2.5) and using (2.8) we get families of analytical solutions for $(2.2)$.

\section{Problems}

In this section, solutions for the targeted fractional-order biological populations models are presented.

\subsection{Problem 1}

Consider the first biological population model given in (1.2):

$$
v_{t}^{\sigma}(t, x, y)=v_{x x}^{2}(t, x, y)+v_{y y}^{2}(t, x, y)+h\left(v^{2}-r\right),
$$

applying the transformation of the form

$$
v(t, x, y)=v(\varphi), \quad \text { where } \quad \varphi=\frac{c t}{\Gamma(\sigma+1)}+k x+l y
$$

the following non-linear ODE is yielded,

$$
c V^{\prime}-h\left(V^{2}-r\right)=0
$$

Balancing $c V^{\prime}$ with $V^{2}$ with the help of (2.6) we get $n=1$.

\subsection{Application of extended $\left(\frac{G^{\prime}}{G}\right)$-expansion method}

Putting $n=1$ in equation (2.4) we get the following $\frac{G^{\prime}}{G}$ for equation (3.3):

$$
V(\varphi)=c_{-1}\left(\frac{G^{\prime}(\varphi)}{G(\varphi)}\right)^{-1}+c_{0}+c_{1}\left(\frac{G^{\prime}(\varphi)}{G(\varphi)}\right)^{1}
$$

where $c_{-1}, c_{0}$ and $c_{1}$ are constants to be determined. Now putting equation (3.4) into equation (3.3) with the help of equation (2.7) we get a polynomial in $\frac{G^{\prime}(\varphi)}{G(\varphi)}$. By coefficient comparison in the polynomial we get a system of algebraic equations. Solving this system with the help of Maple for $c_{-1}, c_{0}, c_{1}, c, h, k$ and $r$ we get the following cases of solutions: 


\section{Case 1:}

$$
c_{0}=c_{0}, c_{1}=0, c_{-1}=\frac{2 \mu c_{0}}{\lambda}, c=c, h=\frac{c \lambda}{2 c_{0}}, r=\frac{D c_{0}^{2}}{\lambda^{2}}, k=i l
$$

\section{Case 2:}

$$
c_{0}=\frac{c_{1} \lambda}{2}, c_{1}=c_{1}, c_{-1}=0, c=-h c_{1}, h=h, r=c_{1}^{2}\left(\frac{\lambda^{2}}{4}-\mu\right), k=i l,
$$

\section{Case 3:}

$$
c_{0}=c_{0}, c_{1}=c_{1}, c_{-1}=c_{-1}, c=h=0, r=r, k=i l,
$$

where $i=\sqrt{-1}$ in above cases.

Now considering case 1 , we have obtained the following families of travelling wave solutions.

Family 1: When $D<0$ then equation (3.4) and equation (2.8) intimate the following solutions: (i) When $C_{1} \neq 0$ and $C_{2}=0$

$$
V_{1}=c_{0}+\frac{2 \mu c_{0}}{\lambda}\left(\frac{\sqrt{-D}}{2} \tan \left(\frac{\sqrt{-D}}{2} \varphi\right)-\frac{A \lambda}{2}\right)^{-1}
$$

(ii) When $C_{1}=0$ and $C_{2} \neq 0$

$$
V_{2}=c_{0}+\frac{2 \mu c_{0}}{\lambda}\left(\frac{\sqrt{-D}}{2} \cot \left(\frac{\sqrt{-D}}{2} \varphi\right)-\frac{\lambda}{2}\right)^{-1}
$$

where $\varphi=\frac{c t}{\Gamma(\sigma+1)}+k x+l y$.

Family 2: When $D>0$ then equation (3.4) and equation (2.8) intimate the following solutions: (i) When $C_{1} \neq 0$ and $C_{2}=0$

$$
V_{3}=c_{0}+\frac{2 \mu c_{0}}{\lambda}\left(\frac{\sqrt{D}}{2} \tanh \left(\frac{\sqrt{D}}{2} \varphi\right)-\frac{\lambda}{2}\right)^{-1}
$$

(ii) When $C_{1}=0$ and $C_{2} \neq 0$

$$
V_{4}=c_{0}+\frac{2 \mu c_{0}}{\lambda}\left(\frac{\sqrt{D}}{2} \operatorname{coth}\left(\frac{\sqrt{D}}{2} \varphi\right)-\frac{\lambda}{2}\right)^{-1}
$$

where $\varphi=\frac{c t}{\Gamma(\sigma+1)}+k x+l y$. 
Family 3: When $D=0$ then equation (3.4) and equation (2.8) intimate the following solutions:

(i) When $C_{1} \neq 0$ and $C_{2}=0$

$$
V_{5}=c_{0}+\frac{2 \mu c_{0}}{\lambda}\left(-\frac{\lambda}{2}\right)^{-1}
$$

(ii) When $C_{1}=0$ and $C_{2} \neq 0$

$$
V_{6}=c_{0}+\frac{2 \mu c_{0}}{\lambda}\left(\frac{1}{\varphi}-\frac{\lambda}{2}\right)^{-1}
$$

where $\varphi=\frac{c t}{\Gamma(\sigma+1)}+k x+l y$.

Now considering Case 2 we have obtained the following families of travelling wave solutions.

Family 4: When $D<0$ then equation (3.4) and equation (2.8) intimate the following solutions: (i) When $C_{1} \neq 0$ and $C_{2}=0$

$$
V_{7}=\frac{c_{1} \lambda}{2}+c_{1}\left(\frac{\sqrt{-D}}{2} \tan \left(\frac{\sqrt{-D}}{2} \varphi\right)-\frac{A \lambda}{2}\right)^{1},
$$

(ii) When $C_{1}=0$ and $C_{2} \neq 0$

$$
V_{8}=\frac{c_{1} \lambda}{2}+c_{1}\left(\frac{\sqrt{-D}}{2} \cot \left(\frac{\sqrt{-D}}{2} \varphi\right)-\frac{\lambda}{2}\right)^{1},
$$

where $\varphi=\frac{-h c_{1} t}{\Gamma(\sigma+1)}+k x+l y$.

Family 5: When $D>0$ then equation (3.4) and equation (2.8) intimate the following solutions:

(i) When $C_{1} \neq 0$ and $C_{2}=0$

$$
V_{9}=\frac{c_{1} \lambda}{2}+c_{1}\left(\frac{\sqrt{D}}{2} \tanh \left(\frac{\sqrt{D}}{2} \varphi\right)-\frac{\lambda}{2}\right)^{1},
$$

(ii) When $C_{1}=0$ and $C_{2} \neq 0$

$$
V_{10}=\frac{c_{1} \lambda}{2}+c_{1} \lambda\left(\frac{\sqrt{D}}{2} \operatorname{coth}\left(\frac{\sqrt{D}}{2} \varphi\right)-\frac{\lambda}{2}\right)^{1}
$$

where $\varphi=\frac{-h c_{1} t}{\Gamma(\sigma+1)}+k x+l y$.

Family 6: When $D=0$ then equation (3.4) and equation (2.8) intimate the following solutions: (i) When $C_{1} \neq 0$ and $C_{2}=0$

$$
V_{11}=\frac{c_{1} \lambda}{2}+c_{1}\left(-\frac{\lambda}{2}\right)^{1}
$$


(ii) When $C_{1}=0$ and $C_{2} \neq 0$

$$
V_{12}=\frac{c_{1} \lambda}{2}+c_{1} \lambda\left(\frac{1}{\varphi}-\frac{\lambda}{2}\right)^{1}
$$

where $\varphi=\frac{-h c_{1} t}{\Gamma(\sigma+1)}+k x+l y$.

Now considering Case 3 we have obtained the following families of travelling wave solutions.

Family 7: When $D<0$ then equation (3.4) and equation (2.8) intimate the following solutions:

(i) When $C_{1} \neq 0$ and $C_{2}=0$

$$
V_{13}=c_{-1}\left(\frac{\sqrt{-D}}{2} \tan \left(\frac{\sqrt{-D}}{2} \varphi\right)+\frac{\lambda}{2}\right)^{-1}+c_{0}+c_{1}\left(\frac{\sqrt{-D}}{2} \tan \left(\frac{\sqrt{-D}}{2} \varphi\right)+\frac{\lambda}{2}\right),
$$

(ii) When $C_{1}=0$ and $C_{2} \neq 0$,

$$
V_{14}=c_{-1}\left(\frac{\sqrt{-D}}{2} \cot \left(\frac{\sqrt{-D}}{2} \varphi\right)+\frac{\lambda}{2}\right)^{-1}+c_{0}+c_{1}\left(\frac{\sqrt{-D}}{2} \cot \left(\frac{\sqrt{-D}}{2} \varphi\right)+\frac{\lambda}{2}\right)
$$

where $\varphi=k x+l y$.

Family 8: When $D>0$ then equation (3.4) and equation (2.8) intimate the following solutions: (i) When $C_{1} \neq 0$ and $C_{2}=0$

$$
V_{15}=c_{-1}\left(\frac{\sqrt{D}}{2} \tanh \left(\frac{\sqrt{D}}{2} \varphi\right)+\frac{\lambda}{2}\right)^{-1}+c_{0}+c_{1}\left(\frac{\sqrt{-D}}{2} \tanh \left(\frac{\sqrt{D}}{2}(\varphi)\right)+\frac{\lambda}{2}\right),
$$

(ii) When $C_{1}=0$ and $C_{2} \neq 0$,

$$
V_{16}=c_{-1}\left(\frac{\sqrt{D}}{2} \operatorname{coth}\left(\frac{\sqrt{D}}{2} \varphi\right)+\frac{\lambda}{2}\right)^{-1}+c_{0}+c_{1}\left(\frac{\sqrt{D}}{2} \operatorname{coth}\left(\frac{\sqrt{D}}{2}(\varphi)\right)+\frac{\lambda}{2}\right),
$$

where $\varphi=k x+l y$.

Family 9: When $D=0$ then equation (3.4) and equation (2.8) intimate the following solutions: (i) When $C_{1} \neq 0$ and $C_{2}=0$

$$
V_{17}=c_{-1}\left(\frac{\lambda}{2}\right)^{-1}+c_{0}+c_{1}\left(\frac{\lambda}{2}\right)
$$

(ii) When $C_{1}=0$ and $C_{2} \neq 0$,

$$
V_{18}=c_{-1}\left(\frac{1}{\varphi}-\frac{\lambda}{2}\right)^{-1}+c_{0}+c_{1}\left(\frac{1}{\varphi}-\frac{\lambda}{2}\right)
$$

where $\varphi=k x+l y$. 


\subsection{Application of Generalized $\left(\frac{G^{\prime}}{G}\right)$-expansion method}

Putting $n=1$ in equation (2.5) we get the following $\frac{G^{\prime}}{G}$ for equation (3.3):

$$
V(\varphi)=c_{-1}\left(d+\frac{G^{\prime}(\varphi)}{G(\varphi)}\right)^{-1}+c_{0}+c_{1}\left(d+\frac{G^{\prime}(\varphi)}{G(\varphi)}\right)^{1}
$$

where $c_{-1}, c_{0}, d$ and $c_{1}$ are constants to be determined. Now putting equation (3.26) into equation (3.3) with the help of equation (2.7) we get a polynomial in $\frac{G^{\prime}(\varphi)}{G(\varphi)}$. By coefficient comparison in the polynomial we get a system of algebraic equations. Solving this system with the help of Maple for $c_{-1}, c_{0}, c_{1}, c, h, l, k$ and $r$ we get the following cases of solutions:

\section{Case 1:}

$$
\begin{aligned}
& c_{0}=-\frac{c_{-1}(\lambda-2 d)}{-\mu+d \lambda-d^{2}}, c_{1}=0, c_{-1}=c_{-1}, c=c, d=d, \\
& h=\frac{-c\left(-\mu+d \lambda-d^{2}\right)}{c_{-1}}, r=\frac{D c_{-1}^{2}}{4\left(-\mu+d \lambda-d^{2}\right)^{2}}, k=i l,
\end{aligned}
$$

\section{Case 2:}

$$
\begin{aligned}
& c_{0}=-c_{1} d+\frac{c_{1} \lambda}{2}, c_{1}=c_{1}, c_{-1}=0, c=-h c_{1}, h=h, r=c_{1}^{2} \frac{\lambda^{2}-\mu}{4} \\
& d=d, k=i l
\end{aligned}
$$

\section{Case 3:}

$$
c_{0}=c_{0}, c_{1}=c_{1}, c_{-1}=c_{-1}, d=d, c=h=0, r=r, k=i l
$$

\section{Case 4:}

$$
\begin{aligned}
& c_{0}=0, c_{1}=c_{1}, c_{-1}=-\mu c_{1}+\frac{\lambda^{2} c_{1}}{4}, d=d, c=-h c_{1}, h=h, r=c_{1}^{2} D \\
& l=\frac{\lambda}{2}, k=i l
\end{aligned}
$$

where $i=\sqrt{-1}$ in above cases.

Now considering Case 1 we have obtained the following families of travelling wave solutions.

Family 1: When $D<0$ then equation (3.4) and equation (2.8) intimate the following solutions:

(i) When $C_{1} \neq 0$ and $C_{2}=0$

$$
V_{1}=-\frac{c_{-1}(\lambda-2 d)}{-\mu+d \lambda-d^{2}}+c_{-1}\left(d+\frac{\sqrt{-D}}{2} \tan \left(\frac{\sqrt{-D}}{2} \varphi\right)-\frac{A \lambda}{2}\right)^{-1}
$$


(ii) When $C_{1}=0$ and $C_{2} \neq 0$

$$
V_{2}=-\frac{c_{-1}(\lambda-2 d)}{-\mu+d \lambda-d^{2}}+c_{-1} d+\left(\frac{\sqrt{-D}}{2} \cot \left(\frac{\sqrt{-D}}{2} \varphi\right)-\frac{\lambda}{2}\right)^{-1}
$$

where $\varphi=\frac{c t}{\Gamma(\sigma+1)}+k x+l y$.

Family 2: When $D>0$ then equation (3.4) and equation (2.8) intimate the following solutions:

(i) When $C_{1} \neq 0$ and $C_{2}=0$

$$
V_{3}=-\frac{c_{-1}(\lambda-2 d)}{-\mu+d \lambda-d^{2}}+c_{-1}\left(d+\frac{\sqrt{D}}{2} \tanh \left(\frac{\sqrt{D}}{2} \varphi\right)-\frac{\lambda}{2}\right)^{-1}
$$

(ii) When $C_{1}=0$ and $C_{2} \neq 0$

$$
V_{4}=-\frac{c_{-1}(\lambda-2 d)}{-\mu+d \lambda-d^{2}}+c_{-1}\left(d+\frac{\sqrt{D}}{2} \operatorname{coth}\left(\frac{\sqrt{D}}{2} \varphi\right)-\frac{\lambda}{2}\right)^{-1}
$$

where $\varphi=\frac{c t}{\Gamma(\sigma+1)}+k x+l y$.

Family 3: When $D=0$ then equation (3.4) and equation (2.8) intimate the following solutions: (i) When $C_{1} \neq 0$ and $C_{2}=0$

$$
V_{5}=-\frac{c_{-1}(\lambda-2 d)}{-\mu+d \lambda-d^{2}}+c_{-1}\left(d-\frac{\lambda}{2}\right)^{-1}
$$

(ii) When $C_{1}=0$ and $C_{2} \neq 0$

$$
V_{6}=-\frac{c_{-1}(\lambda-2 d)}{-\mu+d \lambda-d^{2}}+c_{-1}\left(d+\frac{1}{\varphi}-\frac{\lambda}{2}\right)^{-1},
$$

where $\varphi=\frac{c t}{\Gamma(\sigma+1)}+k x+l y$.

Now considering Case 2 we have obtained the following families of travelling wave solutions.

Family 4: When $D<0$ then equation (3.4) and equation (2.8) intimate the following solutions: (i) When $C_{1} \neq 0$ and $C_{2}=0$

$$
V_{7}=-c_{1} d+\frac{c_{1} \lambda}{2}+c_{1}\left(d+\frac{\sqrt{-D}}{2} \tan \left(\frac{\sqrt{-D}}{2} \varphi\right)-\frac{A \lambda}{2}\right)^{1}
$$

(ii) When $C_{1}=0$ and $C_{2} \neq 0$

$$
V_{8}=-c_{1} d+\frac{c_{1} \lambda}{2}+c_{1}\left(d+\frac{\sqrt{-D}}{2} \cot \left(\frac{\sqrt{-D}}{2} \varphi\right)-\frac{\lambda}{2}\right)^{1}
$$


where $\varphi=\frac{-h c_{1} t}{\Gamma(\sigma+1)}+k x+l y$.

Family 5: When $D>0$ then equation (3.4) and equation (2.8) intimate the following solutions:

(i) When $C_{1} \neq 0$ and $C_{2}=0$

$$
V_{9}=-c_{1} d+\frac{c_{1} \lambda}{2}+c_{1}\left(d+\frac{\sqrt{D}}{2} \tanh \left(\frac{\sqrt{D}}{2} \varphi\right)-\frac{\lambda}{2}\right)^{1},
$$

(ii) When $C_{1}=0$ and $C_{2} \neq 0$

$$
V_{10}=-c_{1} d+\frac{c_{1} \lambda}{2}+c_{1} \lambda\left(d+\frac{\sqrt{D}}{2} \operatorname{coth}\left(\frac{\sqrt{D}}{2} \varphi\right)-\frac{\lambda}{2}\right)^{1},
$$

where $\varphi=\frac{-h c_{1} t}{\Gamma(\sigma+1)}+k x+l y$.

Family 6: When $D=0$ then equation (3.4) and equation (2.8) intimate the following solutions:

(i) When $C_{1} \neq 0$ and $C_{2}=0$

$$
V_{11}=-c_{1} d+\frac{c_{1} \lambda}{2}+c_{1}\left(d-\frac{\lambda}{2}\right)^{1}
$$

(ii) When $C_{1}=0$ and $C_{2} \neq 0$

$$
V_{12}=-c_{1} d+\frac{c_{1} \lambda}{2}+c_{1} \lambda\left(d+\frac{1}{\varphi}-\frac{\lambda}{2}\right)^{1}
$$

where $\varphi=\frac{-h c_{1} t}{\Gamma(\sigma+1)}+k x+l y$.

Now considering Case 3 we have obtained the following families of travelling wave solutions.

Family 7: When $D<0$ then equation (3.4) and equation (2.8) intimate the following solutions: (i) When $C_{1} \neq 0$ and $C_{2}=0$

$$
\begin{aligned}
& V_{13}=c_{-1}\left(d+\frac{\sqrt{-D}}{2} \tan \left(\frac{\sqrt{-D}}{2} \varphi\right)+\frac{\lambda}{2}\right)^{-1}+c_{0}+c_{1}\left(d+\frac{\sqrt{-D}}{2}\right. \\
& \left.\tan \left(\frac{\sqrt{-D}}{2} \varphi\right)+\frac{\lambda}{2}\right),
\end{aligned}
$$

(ii) When $C_{1}=0$ and $C_{2} \neq 0$

$$
\begin{aligned}
& V_{14}=c_{-1}\left(d+\frac{\sqrt{-D}}{2} \cot \left(\frac{\sqrt{-D}}{2} \varphi\right)+\frac{\lambda}{2}\right)^{-1}+c_{0}+c_{1}\left(d+\frac{\sqrt{-D}}{2}\right. \\
& \left.\cot \left(\frac{\sqrt{-D}}{2} \varphi\right)+\frac{\lambda}{2}\right),
\end{aligned}
$$

where $\varphi=k x+l y$. 
Family 8: When $D>0$ then equation (3.4) and equation (2.8) intimate the following solutions: (i) When $C_{1} \neq 0$ and $C_{2}=0$

$$
V_{15}=c_{-1}\left(d+\frac{\sqrt{D}}{2} \tanh \left(\frac{\sqrt{D}}{2} \varphi\right)+\frac{\lambda}{2}\right)^{-1}+c_{0}+c_{1}\left(d+\frac{\sqrt{-D}}{2} \tanh \left(\frac{\sqrt{D}}{2}(\varphi)\right)+\frac{\lambda}{2}\right)
$$

(ii) When $C_{1}=0$ and $C_{2} \neq 0$

$$
V_{16}=c_{-1}\left(d+\frac{\sqrt{D}}{2} \operatorname{coth}\left(\frac{\sqrt{D}}{2} \varphi\right)+\frac{\lambda}{2}\right)^{-1}+c_{0}+c_{1}\left(d+\frac{\sqrt{D}}{2} \operatorname{coth}\left(\frac{\sqrt{D}}{2}(\varphi)\right)+\frac{\lambda}{2}\right),
$$

where $\varphi=k x+l y$.

Family 9: When $D=0$ then equation (3.4) and equation (2.8) intimate the following solutions: (i) When $C_{1} \neq 0$ and $C_{2}=0$

$$
V_{17}=c_{-1}\left(d+\frac{\lambda}{2}\right)^{-1}+c_{0}+c_{1}\left(d+\frac{\lambda}{2}\right)
$$

(ii) When $C_{1}=0$ and $C_{2} \neq 0$

$$
V_{18}=c_{-1}\left(d+\frac{1}{\varphi}-\frac{\lambda}{2}\right)^{-1}+c_{0}+c_{1}\left(d+\frac{1}{\varphi}-\frac{\lambda}{2}\right)
$$

where $\varphi=k x+l y$.

Now considering Case 4 we have obtained the following families of travelling wave solutions.

Family 10: When $D<0$ then equation (3.4) and equation (2.8) intimate the following solutions:

(i) When $C_{1} \neq 0$ and $C_{2}=0$

$$
\begin{aligned}
V_{19}= & \left(-\mu c_{1}+\frac{\lambda^{2} c_{1}}{4}\right)\left(d+\frac{\sqrt{-D}}{2} \tan \left(\frac{\sqrt{-D}}{2} \varphi\right)+\frac{\lambda}{2}\right)^{-1} \\
& +c_{1}\left(d+\frac{\sqrt{-D}}{2} \tan \left(\frac{\sqrt{-D}}{2} \varphi\right)+\frac{\lambda}{2}\right)
\end{aligned}
$$

(ii) When $C_{1}=0$ and $C_{2} \neq 0$

$$
\begin{aligned}
V_{20}= & \left(-\mu c_{1}+\frac{\lambda^{2} c_{1}}{4}\right)\left(d+\frac{\sqrt{-D}}{2} \cot \left(\frac{\sqrt{-D}}{2} \varphi\right)+\frac{\lambda}{2}\right)^{-1} \\
& +c_{1}\left(d+\frac{\sqrt{-D}}{2} \cot \left(\frac{\sqrt{-D}}{2} \varphi\right)+\frac{\lambda}{2}\right)
\end{aligned}
$$

where $\varphi=\frac{-h c_{1} t}{\Gamma(\sigma+1)}+k x+l y$. 
Family 11: When $D>0$ then equation (3.4) and equation (2.8) intimate the following solutions: (i) When $C_{1} \neq 0$ and $C_{2}=0$

$$
\begin{aligned}
V_{21}= & \left(-\mu c_{1}+\frac{\lambda^{2} c_{1}}{4}\right)\left(d+\frac{\sqrt{D}}{2} \tanh \left(\frac{\sqrt{D}}{2} \varphi\right)+\frac{\lambda}{2}\right)^{-1} \\
& +c_{1}\left(d+\frac{\sqrt{-D}}{2} \tanh \left(\frac{\sqrt{D}}{2}(\varphi)\right)+\frac{\lambda}{2}\right)
\end{aligned}
$$

(ii) When $C_{1}=0$ and $C_{2} \neq 0$

$$
\begin{aligned}
V_{22}= & \left(-\mu c_{1}+\frac{\lambda^{2} c_{1}}{4}\right)\left(d+\frac{\sqrt{D}}{2} \operatorname{coth}\left(\frac{\sqrt{D}}{2} \varphi\right)+\frac{\lambda}{2}\right)^{-1} \\
& +c_{1}\left(d+\frac{\sqrt{D}}{2} \operatorname{coth}\left(\frac{\sqrt{D}}{2}(\varphi)\right)+\frac{\lambda}{2}\right)
\end{aligned}
$$

where $\varphi=\frac{-h c_{1} t}{\Gamma(\sigma+1)}+k x+l y$.

Family 12: When $D=0$ then equation (3.4) and equation (2.8) intimate the following solutions: (i) When $C_{1} \neq 0$ and $C_{2}=0$

$$
V_{23}=\left(-\mu c_{1}+\frac{\lambda^{2} c_{1}}{4}\right)\left(d+\frac{\lambda}{2}\right)^{-1}+c_{1}\left(d+\frac{\lambda}{2}\right)
$$

(ii) When $C_{1}=0$ and $C_{2} \neq 0$

$$
V_{24}=-\left(\mu c_{1}+\frac{\lambda^{2} c_{1}}{4}\right)\left(d+\frac{1}{\varphi}-\frac{\lambda}{2}\right)^{-1}+c_{1}\left(d+\frac{1}{\varphi}-\frac{\lambda}{2}\right),
$$

where $\varphi=\frac{-h c_{1} t}{\Gamma(\sigma+1)}+k x+l y$.

\subsection{Problem 2}

Consider the second biological population model given in equation (1.3):

$$
v_{t}^{\sigma}(t, x, y)=v_{x x}^{2}(t, x, y)+v_{y y}^{2}(t, x, y)+v(1-r v),
$$

applying the transformation of the form

$$
v(t, x, y)=v(\varphi) \quad \text { where } \quad \varphi=\frac{c t}{\Gamma(\sigma+1)}+k x+l y
$$

the following non-linear ODE is yielded,

$$
c V^{\prime}-V(1-r V)=0
$$


Balancing $c V^{\prime}$ with $V^{2}$ with the help of equation (2.6) we get $n=1$.

\subsection{Application of extended $\left(\frac{G^{\prime}}{G}\right)$-expansion method}

Putting $n=1$ in equation (2.4) we get the following $\frac{G^{\prime}}{G}$ for equation (3.57):

$$
V(\varphi)=c_{-1}\left(\frac{G^{\prime}(\varphi)}{G(\varphi)}\right)^{-1}+c_{0}+c_{1}\left(\frac{G^{\prime}(\varphi)}{G(\varphi)}\right)^{1}
$$

where $c_{-1}, c_{0}$ and $c_{1}$ are constants to be determined. Now putting equation (3.58) into equation (3.57) with the help of equation (2.7) we get a polynomial in $\frac{G^{\prime}(\varphi)}{G(\varphi)}$. By coefficient comparison in the polynomial we get a system of algebraic equations. Solving this system with the help of Maple for $c_{-1}, c_{0}, c_{1}, c, \lambda, k$ and $\mu$ we get the following cases of solutions:

\section{Case 1:}

$$
\begin{aligned}
& c_{0}=c_{0}, c_{1}=0, c_{-1}=c_{-1}, c=-\frac{c_{0}\left(-1+c_{0} r\right)}{c_{-1}}, \lambda=\frac{c_{-1}\left(-1+2 c_{0} r\right)}{c_{0}\left(-1+c_{0} r\right)}, \\
& \mu=\frac{c_{-1}^{2} r}{c_{0}\left(-1+c_{0} r\right)}, k=i l .
\end{aligned}
$$

\section{Case 2:}

$$
\begin{aligned}
& c_{0}=c_{0}, c_{1}=c_{1}, c_{-1}=0, c=c_{1} r, r \neq 0, \lambda=\frac{-1+2 c_{0} r}{c}, \mu=\frac{c_{0}\left(-1+c_{0} r\right)}{c_{1}^{2} r} \\
& k=i l
\end{aligned}
$$

\section{Case 3:}

$$
c_{0}=\frac{1}{2 r}, c_{1}=\frac{1}{16 c_{-1} r^{2}}, c_{-1}=c_{-1}, c=\frac{1}{16 c_{-1} r}, \lambda=0, \mu=-16 r^{2}, r \neq 0
$$

where $i=\sqrt{-1}$ in above cases.

Now considering Case 1 we have obtained the following families of travelling wave solutions.

Family 1: When $D<0$ then then equation (3.58) and equation (2.8) intimate the following solutions: (i) When $C_{1} \neq 0$ and $C_{2}=0$

$$
V_{1}=c_{0}+c_{-1}\left(\frac{\sqrt{-D}}{2} \tan \left(\frac{\sqrt{-D}}{2} \varphi\right)-\frac{A \lambda}{2}\right)^{-1}
$$

(ii) When $C_{1}=0$ and $C_{2} \neq 0$

$$
V_{2}=c_{0}+c_{-1}\left(\frac{\sqrt{-D}}{2} \cot \left(\frac{\sqrt{-D}}{2} \varphi\right)-\frac{\lambda}{2}\right)^{-1}
$$


where $\varphi=\frac{-\frac{c_{0}\left(-1+c_{0} r\right)}{c-1} t}{\Gamma(\sigma+1)}+k x+l y$.

Family 2: When $D>0$ then equation (3.58) and equation (2.8) intimate the following solutions:

(i) When $C_{1} \neq 0$ and $C_{2}=0$

$$
V_{3}=c_{0}+c_{-1}\left(\frac{\sqrt{D}}{2} \tanh \left(\frac{\sqrt{D}}{2} \varphi\right)-\frac{\lambda}{2}\right)^{-1},
$$

(ii) When $C_{1}=0$ and $C_{2} \neq 0$

$$
V_{4}=c_{0}+c_{-1}\left(\frac{\sqrt{D}}{2} \operatorname{coth}\left(\frac{\sqrt{D}}{2} \varphi\right)-\frac{\lambda}{2}\right)^{-1}
$$

where $\varphi=\frac{-\frac{c_{0}\left(-1+c_{0} r\right)}{c_{-1}} t}{\Gamma(\sigma+1)}+k x+l y$.

Family 3: When $D=0$ then equation (3.58) and equation (2.8) intimate the following solutions: (i) When $C_{1} \neq 0$ and $C_{2}=0$

$$
V_{5}=c_{0}+c_{-1}\left(-\frac{\lambda}{2}\right)^{-1}
$$

(ii) When $C_{1}=0$ and $C_{2} \neq 0$

$$
V_{6}=c_{0}+c_{-1}\left(\frac{1}{\varphi}-\frac{\lambda}{2}\right)^{-1}
$$

where $\varphi=\frac{-\frac{c_{0}\left(-1+c_{0} r\right)}{c_{-1}} t}{\Gamma(\sigma+1)}+k x+l y$.

Now considering Case 2 we have obtained the following families of travelling wave solutions.

Family 4: When $D<0$ then equation (3.58) and equation (2.8) intimate the following solutions: (i) When $C_{1} \neq 0$ and $C_{2}=0$

$$
V_{7}=c_{0}+c_{1}\left(\frac{\sqrt{-D}}{2} \tan \left(\frac{\sqrt{-D}}{2} \varphi\right)-\frac{A \lambda}{2}\right)^{1}
$$

(ii) When $C_{1}=0$ and $C_{2} \neq 0$

$$
V_{8}=c_{0}+c_{1}\left(\frac{\sqrt{-D}}{2} \cot \left(\frac{\sqrt{-D}}{2} \varphi\right)-\frac{\lambda}{2}\right)^{1}
$$

where $\varphi=\frac{c_{1} r t}{\Gamma(\sigma+1)}+k x+l y$. 
Family 5: When $D>0$ then equation (3.58) and equation (2.8) intimate the following solutions: (i) When $C_{1} \neq 0$ and $C_{2}=0$

$$
V_{9}=c_{0}+c_{1}\left(\frac{\sqrt{D}}{2} \tanh \left(\frac{\sqrt{D}}{2} \varphi\right)-\frac{\lambda}{2}\right)^{1}
$$

(ii) When $C_{1}=0$ and $C_{2} \neq 0$

$$
V_{10}=c_{0}+c_{1} \lambda\left(\frac{\sqrt{D}}{2} \operatorname{coth}\left(\frac{\sqrt{D}}{2} \varphi\right)-\frac{\lambda}{2}\right)^{1}
$$

where $\varphi=\frac{c_{1} r t}{\Gamma(\sigma+1)}+k x+l y$.

Family 6: When $D=0$ then equation (3.58) and equation (2.8) intimate the following solutions: (i) When $C_{1} \neq 0$ and $C_{2}=0$

$$
V_{11}=c_{0}+c_{1}\left(-\frac{\lambda}{2}\right)^{1}
$$

(ii) When $C_{1}=0$ and $C_{2} \neq 0$

$$
V_{12}=c_{0}+c_{1} \lambda\left(\frac{1}{\varphi}-\frac{\lambda}{2}\right)^{1}
$$

where $\varphi=\frac{c_{1} r t}{\Gamma(\sigma+1)}+k x+l y$.

Now considering Case 3 we have obtained the following families of travelling wave solutions.

Family 7: When $D<0$ then equation (3.58) and equation (2.8) intimate the following solutions: (i) When $C_{1} \neq 0$ and $C_{2}=0$

$$
\begin{aligned}
V_{13}= & c_{-1}\left(\frac{\sqrt{-D}}{2} \tan \left(\frac{\sqrt{-D}}{2} \varphi\right)+\frac{\lambda}{2}\right)^{-1} \\
& +\frac{1}{2 r}+\frac{1}{16 c_{-1} r^{2}}\left(\frac{\sqrt{-D}}{2} \tan \left(\frac{\sqrt{-D}}{2} \varphi\right)+\frac{\lambda}{2}\right),
\end{aligned}
$$

(ii) When $C_{1}=0$ and $C_{2} \neq 0$

$$
\begin{aligned}
V_{14}= & c_{-1}\left(\frac{\sqrt{-D}}{2} \cot \left(\frac{\sqrt{-D}}{2} \varphi\right)+\frac{\lambda}{2}\right)^{-1} \\
& +\frac{1}{2 r}+\frac{1}{16 c_{-1} r^{2}}\left(\frac{\sqrt{-D}}{2} \cot \left(\frac{\sqrt{-D}}{2} \varphi\right)+\frac{\lambda}{2}\right),
\end{aligned}
$$

where $\varphi=\frac{\frac{1}{16 c_{-1} r} t}{\Gamma(\sigma+1)}+k x+l y$. 
Family 8: When $D>0$ then equation (3.58) and equation (2.8) intimate the following solutions: (i) When $C_{1} \neq 0$ and $C_{2}=0$

$$
\begin{aligned}
V_{15}= & c_{-1}\left(\frac{\sqrt{D}}{2} \tanh \left(\frac{\sqrt{D}}{2} \varphi\right)+\frac{\lambda}{2}\right)^{-1} \\
& +\frac{1}{2 r}+\frac{1}{16 c_{-1} r^{2}}\left(\frac{\sqrt{-D}}{2} \tanh \left(\frac{\sqrt{D}}{2}(\varphi)\right)+\frac{\lambda}{2}\right),
\end{aligned}
$$

(ii) When $C_{1}=0$ and $C_{2} \neq 0$

$$
\begin{aligned}
V_{16}= & c_{-1}\left(\frac{\sqrt{D}}{2} \operatorname{coth}\left(\frac{\sqrt{D}}{2} \varphi\right)+\frac{\lambda}{2}\right)^{-1} \\
& +\frac{1}{2 r}+\frac{1}{16 c_{-1} r^{2}}\left(\frac{\sqrt{D}}{2} \operatorname{coth}\left(\frac{\sqrt{D}}{2}(\varphi)\right)+\frac{\lambda}{2}\right),
\end{aligned}
$$

where $\varphi=\frac{\frac{1}{16 c_{-1}} t}{\Gamma(\sigma+1)}+k x+l y$.

\subsection{Application of Generalized $\left(\frac{G^{\prime}}{G}\right)$-expansion method}

Putting $n=1$ in equation (2.5) we get the following $\frac{G^{\prime}}{G}$ solution for equation (3.57):

$$
V(\varphi)=c_{-1}\left(d+\frac{G^{\prime}(\varphi)}{G(\varphi)}\right)^{-1}+c_{0}+c_{1}\left(d+\frac{G^{\prime}(\varphi)}{G(\varphi)}\right)^{1}
$$

where $c_{-1}, c_{0}, d$ and $c_{1}$ are constants to be determined. Now putting equation (3.78) into equation (3.57) with

the help of equation (2.7) we get a polynomial in $\frac{G^{\prime}(\varphi)}{G(\varphi)}$. By coefficient comparison in the polynomial we get a system of algebraic equations. Solving this system with the help of Maple for $c_{-1}, c_{0}, c_{1}, c, \lambda, d, k$ and $\nu$ we get the following cases of solutions:

\section{Case 1:}

$$
\begin{aligned}
& c_{0}=c_{0}, c_{1}=0, c_{-1}=c_{-1}, c=-\frac{c_{0}\left(-1+c_{0} r\right)}{c_{-1}}, d=d \\
& \lambda=\frac{c_{-1}\left(-1+2 c_{0} r\right)}{c_{0}\left(-1+c_{0} r\right)}, \mu=\frac{c_{-1}^{2} r}{c_{0}\left(-1+c_{0} r\right)}, k=i l .
\end{aligned}
$$

\section{Case 2:}

$$
\begin{aligned}
& c_{0}=c_{0}, c_{1}=c_{1}, c_{-1}=0, c=c_{1} r, r \neq 0, d=d \\
& \lambda=\frac{-1+2 c_{0} r}{c}, \mu=\frac{c_{0}\left(-1+c_{0} r\right)}{c_{1}^{2} r}, k=i l .
\end{aligned}
$$




\section{Case 3:}

$$
\begin{aligned}
& c_{0}=\frac{1}{2 r}, c_{1}=\frac{1}{16 c_{-1} r^{2}}, c_{-1}=c_{-1}, c=\frac{1}{16 c_{-1} r} . \\
& \lambda=0, \mu=-16 r^{2}, r \neq 0, d=d
\end{aligned}
$$

where $i=\sqrt{-1}$ in above cases.

Now considering Case 1 we have obtained the following families of travelling wave solutions.

Family 1: When $D<0$ then equation (3.78) and equation (2.8) intimate the following solutions: (i) When $C_{1} \neq 0$ and $C_{2}=0$

$$
V_{1}=c_{0}+c_{-1}\left(d+\frac{\sqrt{-D}}{2} \tan \left(\frac{\sqrt{-D}}{2} \varphi\right)-\frac{A \lambda}{2}\right)^{-1},
$$

(ii) When $C_{1}=0$ and $C_{2} \neq 0$

$$
V_{2}=c_{0}+c_{-1}\left(d+\frac{\sqrt{-D}}{2} \cot \left(\frac{\sqrt{-D}}{2} \varphi\right)-\frac{\lambda}{2}\right)^{-1},
$$

where $\varphi=\frac{-\frac{c_{0}\left(-1+c_{0} r\right)}{c_{-1}} t}{\Gamma(\sigma+1)}+k x+l y$.

Family 2: When $D>0$ then equation (3.78) and equation (2.8) intimate the following solutions:

(i) When $C_{1} \neq 0$ and $C_{2}=0$

$$
V_{3}=c_{0}+c_{-1}\left(d+\frac{\sqrt{D}}{2} \tanh \left(\frac{\sqrt{D}}{2} \varphi\right)-\frac{\lambda}{2}\right)^{-1},
$$

(ii) When $C_{1}=0$ and $C_{2} \neq 0$

$$
V_{4}=c_{0}+c_{-1}\left(d+\frac{\sqrt{D}}{2} \operatorname{coth}\left(\frac{\sqrt{D}}{2} \varphi\right)-\frac{\lambda}{2}\right)^{-1},
$$

where $\varphi=\frac{-\frac{c_{0}\left(-1+c_{0} r\right)}{c-1} t}{\Gamma(\sigma+1)}+k x+l y$.

Family 3: When $D=0$ then equation (3.78) and equation (2.8) intimate the following solutions: (i) When $C_{1} \neq 0$ and $C_{2}=0$

$$
V_{5}=c_{0}+c_{-1}\left(d-\frac{\lambda}{2}\right)^{-1}
$$

(ii) When $C_{1}=0$ and $C_{2} \neq 0$

$$
V_{6}=c_{0}+c_{-1}\left(d+\frac{1}{\varphi}-\frac{\lambda}{2}\right)^{-1}
$$


where $\varphi=\frac{-\frac{c_{0}\left(-1+c_{0} r\right)}{c-1} t}{\Gamma(\sigma+1)}+k x+l y$.

Now considering Case 2 we have obtained the following families of travelling wave solutions.

Family 4: When $D<0$ then equation (3.78) and equation (2.8) intimate the following solutions:

(i) When $C_{1} \neq 0$ and $C_{2}=0$

$$
V_{7}=c_{0}+c_{1}\left(d+\frac{\sqrt{-D}}{2} \tan \left(\frac{\sqrt{-D}}{2} \varphi\right)-\frac{A \lambda}{2}\right)^{1}
$$

(ii) When $C_{1}=0$ and $C_{2} \neq 0$

$$
V_{8}=c_{0}+c_{1}\left(d+\frac{\sqrt{-D}}{2} \cot \left(\frac{\sqrt{-D}}{2} \varphi\right)-\frac{\lambda}{2}\right)^{1}
$$

where $\varphi=\frac{c_{1} r t}{\Gamma(\sigma+1)}+k x+l y$.

Family 5: When $D>0$ then equation (3.78) and equation (2.8) intimate the following solutions:

(i) When $C_{1} \neq 0$ and $C_{2}=0$

$$
V_{9}=c_{0}+c_{1}\left(d+\frac{\sqrt{D}}{2} \tanh \left(\frac{\sqrt{D}}{2} \varphi\right)-\frac{\lambda}{2}\right)^{1}
$$

(ii) When $C_{1}=0$ and $C_{2} \neq 0$

$$
V_{10}=c_{0}+c_{1} \lambda\left(d+\frac{\sqrt{D}}{2} \operatorname{coth}\left(\frac{\sqrt{D}}{2} \varphi\right)-\frac{\lambda}{2}\right)^{1},
$$

where $\varphi=\frac{c_{1} r t}{\Gamma(\sigma+1)}+k x+l y$.

Family 6: When $D=0$ then equation (3.78) and equation (2.8) intimate the following solutions: (i) When $C_{1} \neq 0$ and $C_{2}=0$

$$
V_{11}=c_{0}+c_{1}\left(d-\frac{\lambda}{2}\right)^{1}
$$

(ii) When $C_{1}=0$ and $C_{2} \neq 0$

$$
V_{12}=c_{0}+c_{1} \lambda\left(d+\frac{1}{\varphi}-\frac{\lambda}{2}\right)^{1}
$$

where $\varphi=\frac{c_{1} r t}{\Gamma(\sigma+1)}+k x+l y$.

Now considering Case 3 we have obtained the following families of travelling wave solutions. 
Family 7: When $D<0$ then equation (3.78) and equation (2.8) intimate the following solutions:

(i) When $C_{1} \neq 0$ and $C_{2}=0$

$$
\begin{aligned}
V_{13}= & c_{-1}\left(d+\frac{\sqrt{-D}}{2} \tan \left(\frac{\sqrt{-D}}{2} \varphi\right)+\frac{\lambda}{2}\right)^{-1} \\
& +\frac{1}{2 r}+\frac{1}{16 c_{-1} r^{2}}\left(d+\frac{\sqrt{-D}}{2} \tan \left(\frac{\sqrt{-D}}{2} \varphi\right)+\frac{\lambda}{2}\right),
\end{aligned}
$$

(ii) When $C_{1}=0$ and $C_{2} \neq 0$

$$
\begin{aligned}
V_{14}= & c_{-1}\left(d+\frac{\sqrt{-D}}{2} \cot \left(\frac{\sqrt{-D}}{2} \varphi\right)+\frac{\lambda}{2}\right)^{-1} \\
& +\frac{1}{2 r}+\frac{1}{16 c_{-1} r^{2}}\left(d+\frac{\sqrt{-D}}{2} \cot \left(\frac{\sqrt{-D}}{2} \varphi\right)+\frac{\lambda}{2}\right)
\end{aligned}
$$

where $\varphi=\frac{\frac{1}{16 c_{-1}{ }^{r}} t}{\Gamma(\sigma+1)}+k x+l y$.

Family 8: When $D>0$ then equation (3.78) and equation (2.8) intimate the following solutions: (i) When $C_{1} \neq 0$ and $C_{2}=0$

$$
\begin{aligned}
V_{15}= & c_{-1}\left(d+\frac{\sqrt{D}}{2} \tanh \left(\frac{\sqrt{D}}{2} \varphi\right)+\frac{\lambda}{2}\right)^{-1} \\
& +\frac{1}{2 r}+\frac{1}{16 c_{-1} r^{2}}\left(d+\frac{\sqrt{-D}}{2} \tanh \left(\frac{\sqrt{D}}{2}(\varphi)\right)+\frac{\lambda}{2}\right),
\end{aligned}
$$

(ii) When $C_{1}=0$ and $C_{2} \neq 0$

$$
\begin{aligned}
V_{16}= & c_{-1}\left(d+\frac{\sqrt{D}}{2} \operatorname{coth}\left(\frac{\sqrt{D}}{2} \varphi\right)+\frac{\lambda}{2}\right)^{-1} \\
& +\frac{1}{2 r}+\frac{1}{16 c_{-1} r^{2}}\left(d+\frac{\sqrt{D}}{2} \operatorname{coth}\left(\frac{\sqrt{D}}{2}(\varphi)\right)+\frac{\lambda}{2}\right),
\end{aligned}
$$

where $\varphi=\frac{\frac{1}{16 c^{r}} t}{\Gamma(\sigma+1)}+k x+l y$.

\section{Discussion AND GRAPHS}

Compared to other analytical methods, the $\left(G^{\prime} / G\right)$-expansion method is an efficient analytical technique because it provides several periodic and solitary traveling wave solutions with some parameters without any linearization and discretization processes for solving problems. The proposed method results expose the inner mechanism of physical phenomena and provide distinct exact solutions of different physical structures in nonlinear science. Besides physical applications, the series form solutions of method help the numerical solvers compare the accuracy of their results and assist them in stability analysis. In the proposed method, the balance between linear effect and nonlinearity effect gives rise to solitons. The solitons are solitary waves that propagate with very little energy use and retain their shape and speed after colliding with another such wave. Our proposed method provides three different important families of solitary wave solutions for the problems, such as periodic 


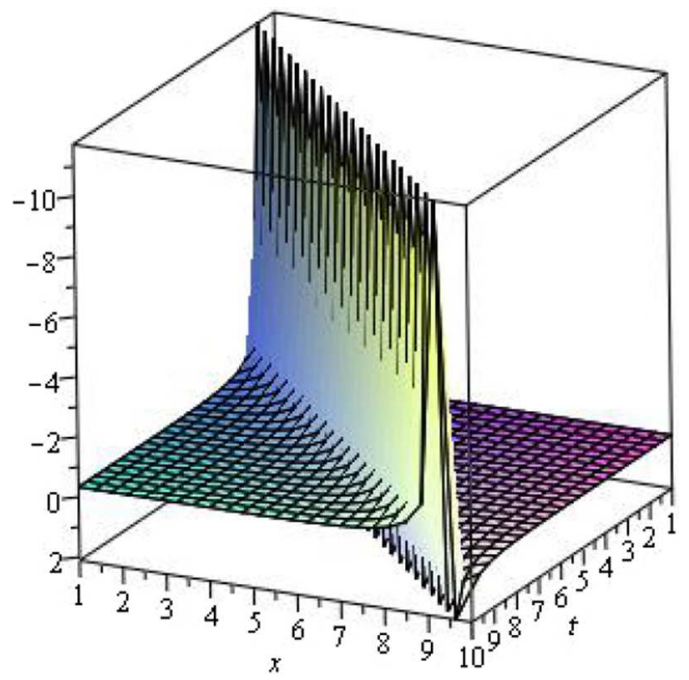

FIgURE 1. Soliton corresponding to hyperbolic solution (3.11) for $\sigma=1, c_{0}=1, \lambda=3, \mu=2$, $k=-1, y=0$ and $c=1$.

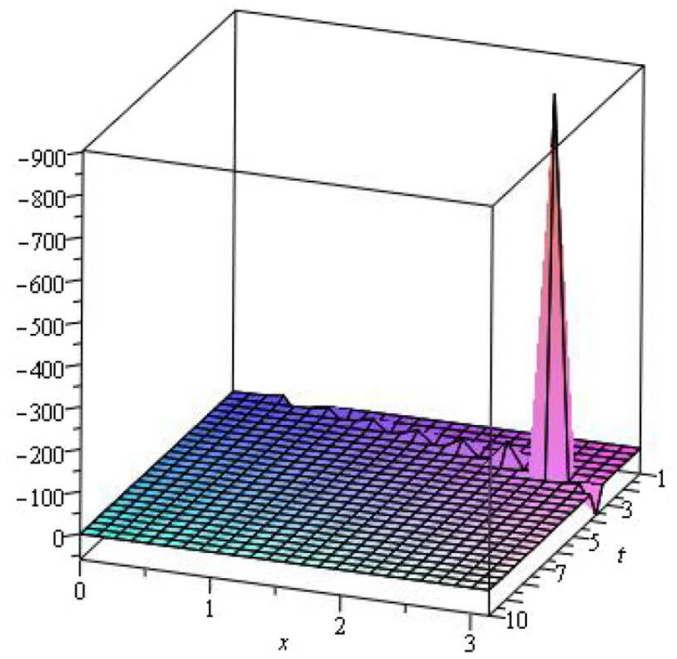

FiguRE 2. Soliton corresponding to rational solution (3.36) for $d=1 \sigma=1, c_{-1}=1, \lambda=6$, $\mu=9, k=-1, y=0$ and $c=1$.

solitary wave solutions when $\lambda^{2}-4 \mu<0$, hyperbolic solitary wave solutions when $\lambda^{2}-4 \mu>0$ and rational solitary wave solutions when $\lambda^{2}-4 \mu=0$. The wave behaviors of some solutions are presented in the Figures 1-3.

Remarks 1: Soliton corresponding to the hyperbolic solution (3.11) represents the topological kink type solitary wave profile.

Remarks 2: Soliton corresponding to the rational solution (3.36) represents dark solution type solitary wave profile.

Remarks 3: Soliton corresponding to the solution (3.8) represents periodic solitary wave profile. 


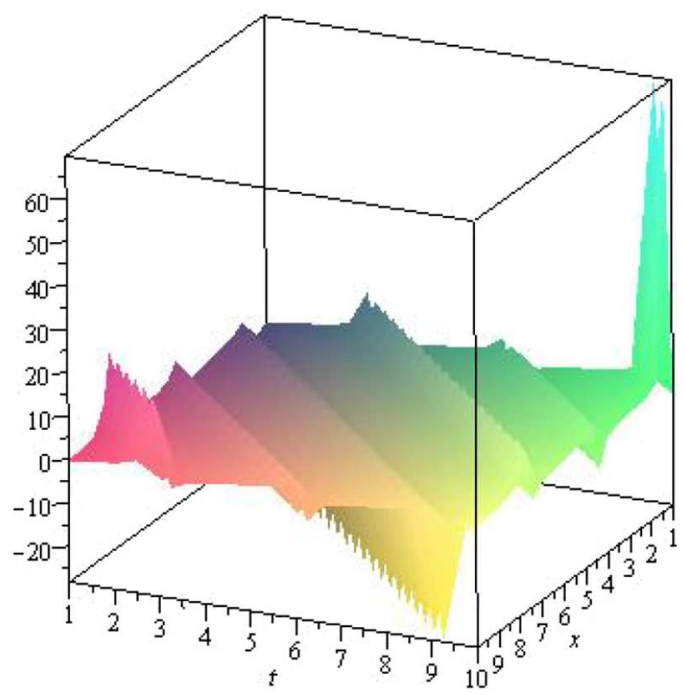

FIGURE 3. Soliton corresponding to periodic solution (3.8) for $\sigma=1, c_{0}=1, \lambda=2, \mu=2$, $k=-1, y=0$ and $c=1$.

\section{Conclusion}

In the current research work, the analytical solution of fractional-order biological population models are solved by using generalized and extended $\left(\frac{G^{\prime}}{G}\right)$-expansion methods. The graphical representation is provided and confirms the validity of the proposed techniques. It has been observed that the geometry of the problems is changed as the different conditions are implemented. As compared to other analytical techniques, the present methods have provided effective and useful dynamics of the given physical phenomena. The three important traveling wave solutions, rational, hyperbolic and trigonometric function solutions, are discussed to analyze different physical phenomena behavior. Furthermore, it is investigated that the present method can be modified to get more families of solutions of the given problem and thus provide a better explanation of the physical problem with the help of free parameter involved in the solution, we can construct solutions that are closely related to the physical problems arising in applied science.

\section{AVAILABILITY OF DATA AND MATERIALS}

Not applicable

\section{COMPETING INTERESTS}

The authors announce that there are not any competing interests.

\section{Authors CONTRIBUtions}

All authors have equally contributed to the manuscript, and read and approved it.

Acknowledgements. José Francisco Gómez Aguilar acknowledges the support provided by CONACyT: Cátedras CONACyT para jóvenes investigadores 2014 and SNI-CONACyT. 


\section{REFERENCES}

[1] M.J. Ablowitz and P.A. Clarkson, Solitons, nonlinear evolution equation and inverse scattering. Cambridge University Press, New York (1991).

[2] D. Baleanu, Y. Ugurlu, M. Inc and B. Kilic, Improved (G'/G)-expansion method for the time-fractional biological population model and Cahn-Hilliard equation. J. Comput. Nonlinear Dyn. 10 (2015) 051016.

[3] A. Bekir and O. Guner, Exact solutions of nonlinear fractional differential equations by (G'/G)-expansion method. Chin. Phys. B 22 (2013) 110202.

[4] Z. Bin, (G'/G)-expansion method for solving fractional partial differential equations in the theory of mathematical physics. Commun. Theor. Phys. 58 (2012) 623.

[5] S. Bushnaq, S. Ali, K. Shah and M. Arif, Exact solution to non-linear biological population model with fractional order. Thermal Sci. 22 (2018) S317-S327.

[6] J.-S. Duan, R. Rach, D. Baleanu and A.-M. Wazwaz, A review of the Adomian decomposition method and its applications to fractional differential equations. Commun. Fract. Calc. 3 (2012) 73-99.

[7] S.A. EL-Wakil, M.A. Madkour and M.A. Abdou, Application of expfunction method for nonlinear evolution equations with variable coefficients. Phys. Lett. A 369 (2007) 62-69.

[8] A.M.A. El-Sayed, S.Z. Rida and A.A.M. Arafa, Exact solutions of fractional-order biological population model. Commun. Theor. Phys. 52 (2009) 992.

[9] R. Hirota, Exact solutions of KdV equation for multiple collisions of solitons. Phys. Rev. Lett. 27 (1971) $1192-1194$.

[10] M.M. Khader and K.M. Saad, A numerical approach for solving the fractional Fisher equation using Chebyshev spectral collocation method. Chaos Solit. Fract. 110 (2018) 169-177.

[11] H. Khan, D. Baleanu, P. Kumam and J.F. Al-Zaidy, Families of travelling waves solutions for fractional-order extended shallow water wave equations, using an innovative analytical method. IEEE Access 7 (2019) 107523-107532.

[12] N.A. Kudryashov, On one of methods for finding exact solutions of nonlinear differential equations. Preprint arXiv:1108.3288v (2011).

[13] D. Kumar, J. Singh and D. Baleanu, On the analysis of vibration equation involving a fractional derivative with Mittag-Leffler law. Math. Methods Appl. Sci. 43 (2020) 443-457.

[14] N.A. Kudryashov, On types of nonlinear non-integrable equations with exact solutions. Phys. Lett. A 155 (1991) $269-275$.

[15] N.A. Kudryashov, Exact solutions of generalized Kuramoto. Sivashinsky equation. Phys. Lett. A 147 (1990) $287-291$.

[16] N.A. Kudryashov and N.B. Loguinova, Extended simplest equation method for nonlinear differential equations. Appl. Math. Comput. 205 (2008) 396-402.

[17] F. Liu, V. Anh and I. Turner, Numerical solution of the space fractional Fokker-Planck equation. J. Comput. Appl. Math. 166 (2004) 209-219.

[18] S. Liu, Z. Fu, S. Liu and Q. Zhao, Jacobi elliptic function expansion method and periodic wave solutions of non linear wave equations. Phys. Lett. A 289 (2001) 69-74.

[19] S. Momani and Z. Odibat, Analytical approach to linear fractional partial differential equations arising in fluid mechanics. Phys. Lett. A 355 (2006) 271-279.

[20] S. Momani and Z. Odibat, Homotopy perturbation method for nonlinear partial differential equations of fractional order. Phys. Lett. A 365 (2007) 345-350.

[21] M.M. Meerschaert and C. Tadjeran, Finite difference approximations for two-sided space-fractional partial differential equations. Appl. Numer. Math. 56 (2006) 80-90.

[22] K.R. Raslan, K.K. Ali and M.A. Shallal, The modified extended tanh method with the Riccati equation for solving the spacetime fractional EW and MEW equations. Chaos Solit. Fractals 103 (2017) 404-409.

[23] K.M. Saad, M. Alqhtani and J.F. Gómez-Aguilar, Fractal-fractional study of the hepatitis C virus infection model. Res. Phys. 19 (2020) 103555.

[24] K.M. Saad, J.F. Gómez-Aguilar and A.A. Almadiy, A fractional numerical study on a chronic hepatitis C virus infection model with immune response. Chaos, Solit. Fract. 139 (2020) 110062.

[25] F. Shakeri and M. Dehghan, Numerical solution of a biological population model using He's variational iteration method. Comput. Math. Appl 54 (2007) 1197-1209.

[26] J. Singh, H. Kamil Jassim and D. Kumar, An efficient computational technique for local fractional Fokker Planck equation. Physica A 555 (2020) 124525.

[27] J. Singh, D. Kumar, D. Baleanu and S. Rathore, On the local fractional wave equation in fractal strings. Math. Methods Appl. Sci. 42 (2019) 1588-1595.

[28] H.M. Srivastava, K.M. Saad, J.F. Gómez-Aguilar and A.A. Almadiy, Some new mathematical models of the fractional-order system of human immune against IAV infection. Math. Biosci. Eng. 17 (2020) 4942-4969.

[29] P. Veeresha, D.G. Prakasha, J. Singh, I. Khan and D. Kumar, Analytical approach for fractional extended Fisher-Kolmogorov equation with Mittag-Leffler kernel. Adv. Differ. Equ. 2020 (2020) 1-17.

[30] M. Wang, X. Li and J. Zhang, The (G'/G)-expansion method and travelling wave solutions of nonlinear evolution equations in mathematical physics. Phys. Lett. A 372 (2008) 417-423.

[31] E.M.E. Zayed and K.A. Gepreel, The (G'/G)-expansion method for finding traveling wave solutions of nonlinear partial differential equations in mathematical physics. J. Math. Phys. 50 (2009) 013502.

[32] E.M.E. Zayed and S. Al-Joudi, Applications of an extended (G'/G)-expansion method to find exact solutions of nonlinear PDEs in mathematical physics. Math. Probl. Eng 2010 (2010). 
[33] Y. Zhang, Solving STO and KD equations with modified Riemann Liouville derivative using improved (G'/G)-expansion function method. IAENG Int. J. Appl. Math. 45 (2015) 16-22.

[34] J. Zhang, F. Jiang and X. Zhao, An improved (G'/G)-expansion method for solving nonlinear evolution equations. Int. J. Comput. Math. 87 (2010) 1716-1725.

[35] P. Zhuang, F. Liu, V. Anh and I. Turner, New solution and analytical techniques of the implicit numerical method for the anomalous subdiffusion equation. SIAM J. Numer. Anal. 46 (2008) 1079-1095. 